\title{
FINITE JUMPS IN MILNOR NUMBER IMPLY VANISHING FOLDS
}

\author{
DONAL B. O'SHEA'
}

\begin{abstract}
Let $\left\{X_{l}\right\}$ be a family of isolated hypersurface singularities in which the Milnor number is not constant. It is proved that there must be a vanishing fold centered at any $t=t_{0}$ at which the Milnor number of the $X$, changes discontinuously. This is much stronger than the condition that the Whitney conditions fail.
\end{abstract}

1. Introduction. The purpose of this note is to establish a useful result concerning the geometric behaviour of families of complex hypersurfaces with an isolated singularity in which the Milnor number is not constant. After establishing more notation we state and prove our result. We close with a number of remarks which the reader may wish to peruse before examining the details of the proof.

2. The main result. Let $h: \mathbf{C}^{n} \rightarrow \mathbf{C}$ be a polynomial with an isolated singularity at the origin $\mathbf{0}$ and suppose that $h(\mathbf{0})=0$. We will assume throughout that $n \geqslant 2$. We let $\mu(h)$ denote the Milnor number of $h$ at the origin (see $[6, \S 6])$. If $R$ and $\delta$ are positive real numbers, set $B_{R}=\left\{x \in \mathbf{C}^{n}:|x| \leqslant R\right\}, S_{R}=\left\{x \in \mathbf{C}^{n}:|x|=R\right\}$ and $D_{\delta}=\{z \in \mathbf{C}:|z| \leqslant \delta\}$. For fixed $z \in \mathbf{C}$ we write $S_{r} \pitchfork h^{-1}(z)$ if $S_{R} \cap h^{-1}(z)$ contains no singular points of $h$ and if the manifold consisting of the smooth points of $h^{-1}(z)$ intersects $S_{R}$ transversally.

Let $r: \mathbf{C}^{n} \rightarrow \mathbf{R}_{+}$be the usual squared distance function $r(x)=|x|^{2}=\left|x_{1}\right|^{2}$ $+\cdots+\left|x_{n}\right|^{2}$ where $x=\left(x_{1}, \ldots, x_{n}\right)$. A point $p \in h^{-1}(0)$ is said to be a kink of $h^{-1}(0)$ if $p$ is not a singular point of $h$ and if $p$ is a critical point of $r$ restricted to the manifold of smooth points of $h^{-1}(0)$. Set $d h(p)=\left.\left(\partial h / \partial x_{1}, \ldots, \partial h / \partial x_{n}\right)\right|_{x=p}$. An easy computation shows that a nonsingular $p \in h^{-1}(0)$ is a kink if and only if $d h(p)=k \vec{p}$ for some $k \in \mathbf{C}-\{0\}$ (here, and in what follows, an overbar denotes complex conjugation). Notice that this means that the set $\left\{x \in \mathbf{C}^{n}: x\right.$ is a kink or a singular point of $\left.h^{-1}(0)\right\}$ can be defined by polynomial equations in $x$ and $\bar{x}$ and is thus a real algebraic subset of $\mathbf{C}^{n}$.

We suppose henceforth that $f(x, t)$ is a polynomial in $x=\left(x_{1}, \ldots, x_{n}\right) \in \mathbf{C}^{n}$ with coefficients that are real analytic complex-valued functions in $t \in[0,1]$. For fixed $t \in[0,1]$ write $f_{t}(x)=f(x, t)$. We suppose further that, for every $t \in[0,1], f_{t}(\mathbf{0})=0$ and $\boldsymbol{O}$ is an isolated critical point of $f_{t}$.

Let $\sigma:[0, \varepsilon] \rightarrow \mathbf{C}^{n} \times[0,1]$ be a real analytic path $\sigma(s)=(x(s), t(s))$ such that:

(1) $\sigma(0)=(0,0)$.

Received by the editors March 15, 1982 and, in revised form. July 12, 1982

1980 Mathematics Subject Classification. Primary 14D05: Secondary 14B07, 32C40, 32G11.

'This research was partially supported by a Faculty Grant from Mount Holyoke College. 
(2) $|x(s)|>0$ and $|t(s)|>0$ for all $0<s \leqslant \varepsilon$, and

(3) $f(x(s), t(s))=0$ for all $0 \leqslant s \leqslant \varepsilon$.

Then $\sigma$ is said to be a nontrivial critical arc (centered at $t=0$ ) of the family $\left\{f_{t}^{-1}(0)\right\}$ if $x(s)$ is a singularity of $f_{t(s)}^{-1}(0)$ for every $s \in[0, \varepsilon]$. The path $\sigma$ will be called a vanishing fold (centered at $t=0$ ) of $\left\{f_{t}^{-1}(0)\right\}$ if $x(s)$ is a kink of $f_{t(s)}^{-1}(0)$ for every $s \in(0, \varepsilon]$.

THEOREM. Let $f(x, t)$ be as above and let $\left\{f_{t}^{-1}(0)\right\}$ be the corresponding family of hypersurfaces. Let $\mu_{t}$ be the Milnor number of $f_{t}$ at the origin and suppose that $\mu_{t} \equiv \mu$ is constant for $0<t \leqslant 1$ and that $\mu_{0}>\mu$. Then, either the family $\left\{f_{t}^{-1}(0)\right\}$ admits a nontrivial critical arc centered at $t=0$ or it has a vanishing fold centered at $t=0$.

Proof. Suppose that $\left\{f_{t}^{-1}(0)\right\}$ has no nontrivial critical arc centered at $t=0$. We shall show that there is a vanishing fold centered at $t=0$. Choose $R>0$ such that $f_{0}$ has no singular points in $B_{R}-\{0\}$ and $S_{R^{\prime}} \pitchfork f_{0}^{-1}(0)$ for all $0<R^{\prime} \leqslant R$ (see [6, §2]). Using the continuity of $\left|d f_{t}(x)\right|$ on $S_{R}$, choose $0<\tau \ll R$ such that $S_{R} \Uparrow f_{t}^{-1}(0)$ and the sets $S_{R}$ and $f_{t}^{-1}(0) \cap\left(B_{R}-\{\mathbf{0}\}\right)$ contain no singular points of $f_{t}$ for every $0 \leqslant t \leqslant \tau$. Finally, choose $\delta>0$ such that $f_{t}^{-1}(z) \Uparrow S_{R}$ for all $0 \leqslant t \leqslant \tau$ and all $z \in D_{\delta}=\{z \in \mathbf{C}:|z| \leqslant \delta\}$.

Let $\Sigma=\left\{(x, t) \in B_{R} \times[0, \tau]: d f_{t}(x)=0\right\}$. It is easy to see that the set $\Sigma$ is real analytic with real dimension equal to one and consists of the line $\{0\} \times[0, \tau]$ together with finitely many one-dimensional arcs meeting $\{0\} \times[0, \tau]$ only at $(0,0)$. Let $H: \mathbf{C}^{n} \times[0,1] \rightarrow \mathbf{C} \times[0,1]$ be the map defined by $H(x, t)=\left(f_{t}(x), t\right)$. Set $\Delta=H(\Sigma) \cap\left(D_{\delta} \times[0, \tau]\right)$ and $\Delta^{c}=\left(D_{\delta} \times[0, \tau]\right)-\Delta$. The set $\Delta^{c}$ is connected. Using an argument similar to Ehresmann [4] we find that the restriction of $H$ to $B_{R} \times[0, \tau] \cap H^{-1}\left(\Delta^{c}\right)$ is the projection map of a smooth fibration with base space $\Delta^{c}$. We conclude that the manifolds $f_{t}^{-1}(z) \cap B_{R}$ are diffeomorphic for $(z, t) \in \Delta^{c}$.

When $z \in D_{\delta}-\{0\}$ the manifold (with boundary) $f_{0}^{-1}(z) \cap B_{R}$ has the homotopy type of a bouquet of $\mu_{0}$ real $(n-1)$-dimensional spheres [6, §§6-7] and, hence, so does $f_{t}^{-1}(z) \cap B_{R}$ for every $(z, t) \in \Delta^{c}$. However (using the results of $[6, \S \S 6-7]$ again), we can choose $R(t)$ where $0<R(t)<R$ and $\delta(t)$ where $0<\delta(t) \leqslant \delta$ such that $f_{t}^{-1}(z) \cap B_{R(t)}$ is a smooth manifold with the homotopy type of $\mu<\mu_{0}$ spheres whenever $(z, t) \in\left(D_{\delta(t)}-\{0\}\right) \times(0, \tau]$. For fixed $t \in(0, \tau]$ we can, upon shrinking $\delta(t)$ if necessary, assume that $f_{t}^{-1}\left(D_{\delta(t)}-\{0\}\right) \cap B_{R}$ contains no critical points of $f_{t}$.

Suppose $(z, t) \in\left(D_{\delta(t)}-\{0\}\right) \times(0, \tau]$ and let $r_{z, t}: f_{t}^{-1}(z) \cap B_{R} \rightarrow[0, R]$ be the restriction of $r: x \rightarrow|x|^{2}$ to $f_{t}^{-1}(z) \cap B_{R}$. Since $f_{t}^{-1}(z) \cap B_{R}$ and $f_{t}^{-1}(z) \cap B_{R(t)}$ have different homotopy types, $r_{z, t}$ must have a critical point in $f_{t}^{-1}(z) \cap\left(B_{R}-B_{R(t)}\right)$ (see, for example, [7, Theorem 3.1]). We claim that $f_{t}^{-1}(0)$ has a kink for each $0<t \leqslant \tau$. To see this, note that if $f_{t}(x)=z$ is such that $d f_{t}(x) \neq 0$ then $x$ is a critical point of $r_{z: t}$ if and only if $d f_{t}(x)=k \bar{x}$ for some $k \in \mathbf{C}-\{0\}$. Thus, for each $t$ the set $A_{t}=\left\{x \in B_{R}: x\right.$ is a critical point of $r_{z, t}$ or $\left.f_{t}\right\}$ is (the restriction of) a real algebraic subset of $\mathrm{C}^{n}$. Since real algebraic sets are closed and since $A_{t} \cap\left(B_{R}-B_{R(t)}\right)$ contains points arbitrarily close to $f_{t}^{-1}(0) \cap\left(B_{R}-B_{R(t)}\right)$ there exists a point $x_{t} \in A_{t}$ $\cap f_{t}^{-1}(0)$ for which $R(t) \leqslant\left|x_{t}\right|<R$. Moreover, by assumption $x_{t}$ is not a critical point of $f_{t}$ and so $x_{t}$ must be a critical point of $r_{0, t}$. That is, $x_{t}$ is a kink of $f_{t}^{-1}(0)$. 
Now observe that we can choose the kinks $x_{t}$ of $f_{t}^{-1}(0)$ so that $x_{t}$ tends to the origin as $t$ tends to 0 . This is clear because we can repeat the entire argument above with smaller and smaller choices of $R$. The set $A=\left\{(x, t) \in\left(B_{R}-\{0\}\right) \times[0, \tau]\right.$ : $f_{t}(x)=0$ and $d f_{t}(x)=k \bar{x}$ for some $\left.k \in \mathbf{C}\right\}$ is clearly real analytic. It consists entirely of kinks (because we are assuming that there is no nontrivial critical arc) and contains points arbitrarily close to $(0,0)$. Applying the curve selection lemma [3] gives us a vanishing fold. This establishes the theorem.

3. Remarks. (1) The theorem (and proof) still hold if each of the $f_{t}$ are analytic functions of $x_{1}, \ldots, x_{n}$. One may also take $t$ to be complex.

(2) Let $\left\{f_{t}^{-1}(0)\right\}$ be as in the statement of the theorem. If we replace the assumption that $\mu_{0}>\mu$ by the assumption that $\mu_{0}=\mu$, then the easy part of the proof of the Lê-Ramanujam theorem [5] shows that "no vanishing folds implies constant topological type". It is an open question as to whether there exist $\mu$-constant families which have a vanishing fold.

(3) The intuitive content of the above theorem is that a jump in the Milnor number can be detected purely geometrically (that is, from an examination of the varieties $\left\{f_{t}^{-1}(0)\right\}$ as $t \rightarrow 0$ ).

(4) The condition that $f_{t}$ have an isolated singularity for all $t$ is crucial. For example, let $f_{t}(x, y)=(x+t y)(x-t y)$. There is no vanishing fold or nontrivial critical arc centered at $t=0$. Yet, the singularity of $f_{0}$ at the origin is not even reduced.

(5) The statement that a family $\left\{f_{t}^{-1}(0)\right\}$ of isolated singularities have a vanishing fold centered at $t=0$ is much stronger than the statement that the pair of strata $\left(f^{-1}(0)-\{t\right.$-axis $\},\{t$-axis $\left.\}\right)$ satisfy the Whitney conditions at $(0,0)$. For example, it is easy to check that a quasihomogeneous polynomial cannot have a kink. Hence, any family of isolated singularities of quasihomogeneous polynomials cannot have a vanishing fold. However, Briançon and Speder [1] have given examples of such families which do not satisfy the Whitney conditions. An interesting application of the theorem of $\$ 2$ can be made in this context. In the case of the Briançon-Speder families, a generic hyperplane section cuts out a family of curves which satisfies the conditions of our theorem. We can, therefore, find a vanishing fold in this family of curves. Considering the vanishing folds as an analytic path in the original family of surfaces gives a path along which the limit condition involved in Whitney condition $b$ is violated. I do not know whether this phenomenon (that is, vanishing folds in hyperplane sections give Whitney faults in the original family) holds in general. If it did, and I suspect that it does, our theorem would yield a simple proof of Briançon and Speder's result [2] that "Whitney conditions imply $\mu^{*}$ constant". (For a definition of $\mu^{*}$ see [9].)

(6) The notion of a kink is highly dependent on the choice of coordinates. Hence, it seems unlikely (but, it is by no means certain) that the existence of vanishing folds is independent of the coordinate choice. (See [8].)

(7) The theorem and proof in $\$ 2$ continue to hold if we replace $r: \mathbf{C}^{n} \rightarrow \mathbf{R}_{+}$in the definition of kink and vanishing fold by a squared distance function with respect to any affine metric on $\mathbf{C}^{n}$. 
(8) A somewhat more detailed version of this paper is available from the author upon request.

\section{REFERENCES}

1. J. Briançon and J. P. Speder, La trivialité topologique n' implique pas les conditions de Whitnev, C. R. Acad. Sci.Paris Sèr. A-B 280 (1975), 365-367.

2. , Les conditions de Whitney impliquent " $\mu^{*}$ constant", Ann. Inst. Fourier (Grenoble) 26 (1976), $153-163$.

3. F. Bruhat and H. Cartan, Sur la structure des sous-ensembles analytiques réels, C. R. Acad. Sci. Paris Sér. A-B 244 (1957), 988-990.

4. C. Ehresmann, Sur les espaces fibrés differentiables, C. R. Acad. Sci. Paris Sèr. A-B 224 (1947), 1611-1612.

5. Lê Dũng Tráng and C. P. Ramanujam. The invariance of Milnor's number implies the invariance of the topological type, Amer. J. Math. 98 (1976), 67-78.

6. J. Milnor, Singular points of complex hypersurfaces, Ann. of Math. Studies, no. 61, Princeton Univ. Press, Princeton, N. J., 1968.

7. Morse theory, Ann. of Math. Studies, no. 51, Princeton Univ. Press, Princeton, N. J., 1969.

8. D. O'Shea, Vanishing folds in $\mu$-constant families, Proc. Sympos. Pure Math.,vol. 40, Amer. Math. Soc., Providence, R. I. (to appear).

9. B. Teissier, Cycles évanescents, sections planes et conditions de Whitney. Singularités à Cargese, Astérisque 7-8 (1973).

Department of Mathematics, Mount Holyoke College, South Hadley, Massachusetts 01075 